\title{
Effect of LIV1 on the sensitivity of ovarian cancer cells to trichostatin A
}

\author{
XIAOLI MA $^{1 *}$, HUA DUAN ${ }^{1 *}$, JIA LIU $^{2}$, QINGQING MO ${ }^{2}$, \\ CHENGJUAN SUN $^{1}$, DING MA ${ }^{2}$ and JIANDONG WANG ${ }^{1}$ \\ ${ }^{1}$ Gynecological Minimal Invasive Center, Beijing Obstetrics and Gynecology Hospital, \\ Capital Medical University, Beijing 100006; ${ }^{2}$ Cancer Biology Research Center, \\ Tongji Hospital, Tongji Medical College, Huazhong University of Science and \\ Technology, Wuhan, Hubei 430030, P.R. China
}

Received July 26, 2014; Accepted October 31, 2014

DOI: $10.3892 /$ or.2014.3622

\begin{abstract}
In a previous study, we used a functional gene screen approach to identify the key genes responsible for the tumor-selective action of trichostatin A (TSA), of which LIV1, a novel zinc transporter, was isolated by its marked ability to confer resistance against TSA-induced apoptosis. The aim of the present study was to investigate the effect of LIV1 expression on the sensitivity of ovarian cancer cells to TSA. We tested the induction of LIV1 in ovarian cancer cells and clinical samples after TSA treatment by real-time PCR and western blot analysis. We investigated the effect of LIV1 expression on the sensitivity of ovarian cancer cells to TSA by MTT assay, flow cytometry and colony forming assays. Finally, we analyzed the mechanism of LIV1 in ovarian cancer cells by western blot analysis. We found that the levels of LIV1 mRNA and protein were significantly upregulated after TSA treatment. The viability and colony forming rates of the ovarian cancer cells transfected with AS-LIV1 (pCEP4 carrying antisense LIV1 cDNA) were obviously higher than the rates of the control as detected by MTT and colony forming assays, which could be reversed by FL-LIV1 (pCEP4 carrying full-length LIV1 cDNA). The apoptotic rate of the AS-LIV1 cells was markedly lower than the rate of the control as determined FACS. Using western blot analysis, we demostrated that the inhibition of TSA-induced apoptosis by knockdown of LIV1 might be associated with decreased endogenous levels of Bcl-2, enhanced levels of Bax and cleavage of procaspase- 3 .
\end{abstract}

Correspondence to: Dr Jiandong Wang, Gynecological Minimal Invasive Center, Beijing Obstetrics and Gynecology Hospital, Capital Medical University, 251 Yao Jiayuan Road, Chaoyang, Beijing 100026, P.R. China

E-mail: wangjiandongxy@hotmail.com

*Contributed equally

Key words: ovarian cancer, drug resistance, LIV1, trichostatin A, apoptosis
The present study suggests that the drug resistance of ovarian cancer cells to TSA may be related to expression of the LIV1 gene, and targeting LIV1 could be exploited as a novel strategy to more effectively kill ovarian cancer cells.

\section{Introduction}

Ovarian cancer is one of the most common gynecological malignancies and is associated with a poor prognosis. Historically, it is considered a 'silent' cancer since most patients present with late-stage disease $(1,2)$. Despite advances in surgery and the development of more effective chemotherapy, ovarian cancer remains the number one cause of death from gynecologic cancer. Drug resistance is the predominant cause of death in late-stage patients. Approximately $30 \%$ of patients whose tumors are platinum-resistant will generally either progress during primary therapy or shortly thereafter. Moreover, there is no preferred standard second-line chemotherapy to offer these patients $(3,4)$. Thus, elucidation of mechanisms and identification of new therapeutic targets and drugs for ovarian cancer are critical to reduce the high mortality.

Histone deacetylase inhibitors (HDACis) show promise as a novel class of anticancer agents in a wide spectrum of tumors including ovarian cancer (5-7). To date, at least 14 HDACis are being tested in over 100 clinical trials and have displayed encouraging therapeutic responses with surprisingly good safety profiles. The clinical potential of HDACis has been well documented by the successful development of vorinostat/SAHA and romidepsin, which have been approved by the U.S. Food and Drug Administration $(5,8,9)$. Despite the rapid progress achieved, clinical data have shown that there is limited efficacy for HDACis as a single agent. Most current clinical trials are combination studies looking at HDACis in combination with other agents $(6,10,11)$. All of these combination trials seek to increase the antitumor activity of the treatments. Although these combination strategies follow a rational molecular approach in some cases, in most instances, they are relatively empirical. Accordingly, synergism in antitumor efficacy might be accompanied by adverse effects that are rarely or never seen with HDACis alone such as severe 
myelosuppression (11). Therefore, revealing the molecular mechanisms underlying the low potency of HDACis is pivotal in determining the optimal application of this class of therapeutic agents in the treatment of ovarian cancer.

Trichostatin A (TSA) is a natural compound and is one of the most potent HDACis. In a previous study, we conducted a functional gene screen approach to identify the key genes responsible for the tumor-selective action of TSA. LIV1 was isolated by its marked ability to confer resistance against TSA-induced apoptosis. Our data preliminarily implied that the inhibition of TSA-induced apoptosis by knockdown of LIV1 might be associated with its ability to disrupt intracellular zinc homeostasis in cervical cancer cells (12). To date, research on LIV1 is very limited. Only several studies have speculated that LIV1 might be related to the poor prognosis of breast cancer and could control epithelial-mesenchymal transition in zebrafish gastrula organizer, which have not been fully verified $(13,14)$. Therefore, the effect of LIV1 on ovarian cancer cells is totally unknown. The present study was designed to explore the effect of LIV1 on the sensitivity of ovarian cancer cells to TSA and to provide a theoretical basis for further clinical targeted therapy.

\section{Materials and methods}

Cells and reagents. The human ovarian cancer cell lines A2780 and SKOV3 were purchased from the American Type Culture Collection and cultured in Dulbecco's modified Eagle's medium (DMEM) containing 10\% FCS. All cells were cultured at $37^{\circ} \mathrm{C}$ in a humidified $5 \% \mathrm{CO}_{2}$ atmosphere. HDACis, TSA and SAHA, were purchased from Sigma and dissolved in DMSO.

Primary ovarian cancer cell culture. Ovarian cancer tissues were obtained from 6 patients hospitalized in the Beijing Obstetrics and Gynecology Hospital affiliated to Capital Medical University before any clinical therapy. All research protocols in the present study were approved by our Ethics Committee, and all patients gave written informed consent to enroll in the study. These patients were 17-75 (mean, 47.1 \pm 13.5 ) years of age and were hospitalized between June 2012 and December 2013; ovarian cancer was confirmed by pathological diagnosis. Four patients were pathologically classified as serous adenocarcinoma and 2 as mucoid adenocarcinoma. According to the Federation International of Gynecology and Obstetrics (FIGO) staging system, 1 case was classified as FIGO stage I, 2 as FIGO stage II, 2 as FIGO stage III, and 1 as FIGO stage IV. Cells were isolated and cultured as previously described (15).

Cell viability assays. Cell viability was determined using an MTT assay. In brief, $5 \times 10^{3}$ cells were plated into each well of 96-well plates at $72 \mathrm{~h}$ after the indicated treatments, after which $5 \mathrm{mg} / \mathrm{ml}$ MTT was added and incubated at $37^{\circ} \mathrm{C}$ for $4 \mathrm{~h}$. Media were then removed, and $1 \mathrm{ml}$ of DMSO was added to solubilize the MTT-formazan product. The MTT absorbance was then measured at $570 \mathrm{~nm}$ on a Multiscan JX ver 1.1 (Thermo Labsystems). Results are expressed as a percentage of the viable cells in the DMSO-treated group. Each data point is the mean \pm SEM of six replicates.
Apoptosis assays. Cells were stained with Annexin V and propidium iodide (PI) and the percentage of apoptotic cells were determined by flow cytometry as previously described (15). CellQuest software was used for data acquisition and analysis.

Real-time PCR. Quantitative PCR was conducted in ABI Prism 7000 using the SYBR-Green PCR Master Mix (Sigma) with the following set of primers: LIV1, 5'-GGT GAT GGC CTG CAC AAT TTC-3' and 5-TTA ACG GTC ATG CCA GCC TTT AGT A-3; 18s RNA, 5'-AGT CCC TGC CCT TTG ACA CA-3' and 5'-GAT CCG AGG GCC TCA CTA AAC-3'. 18s RNA was used as internal control. All primers were designed with the Primer 3 software. A melting curve assay was performed to determine the purity of the amplified product. Contamination with genomic DNA was not detected in any of the analyzed samples. Each sample was assayed in triplicate, analysis of the relative gene expression data used the $2^{-\mathrm{ACT}}$ method (15), and the results are expressed as fold induction compared with the untreated group.

Western blot analysis. Preparation of protein samples and western blotting were carried out as previously described (15). Antibodies against LIV1 were purchased from Novus Biologicals. Antibodies against Bcl-2, Bax, and caspase-3 were purchased from Cell Signaling Technology. Antibodies against $\beta$-actin were purchased from Santa Cruz Biotechnology.

Colony forming assays. A2780 and SKOV3 cells were stably transfected with PCEP4-CAT and AS-LIV1/FL-LIV1 and cultured for $24 \mathrm{~h}$. Cells were then treated with $500 \mathrm{nmol} / 1$ TSA or $500 \mathrm{nmol} / \mathrm{l}$ apicidine for $24 \mathrm{~h}$ and plated in triplicate in 24 -well plates at 50 cells/well. Plates were subsequently incubated for 14 days in a humidified incubator at $37^{\circ} \mathrm{C}$, and colonies were fixed with $4 \%$ paraformaldehyde and stained with $0.5 \%$ crystal violet and counted using a dissecting microscope (magnification, $\mathrm{x} 50$ ). Three random fields were counted for each triplicate of samples, and average values were presented as the means $\pm \mathrm{SD}$.

\section{Results}

TSA induces the expression of LIVI in ovarian cancer cells. To test whether expression of LIV1 is induced by TSA, we investigated the effects of TSA on the mRNA and protein expression of LIV1 in the ovarian cancer cells. A2780 and SKOV3 cells were chosen because they had moderate levels of LIV1. First, cells were treated with $500 \mathrm{nmol} / \mathrm{l} \mathrm{TSA}$ for various lengths of time. As shown in Fig. 1A and B, transcription of LIV1 was highly induced. At $12 \mathrm{~h}$ post-treatment with TSA, transcription induction reached a maximal level $(5.63 \pm 0.80$-fold for A2780; 4.67 \pm 0.51 -fold for SKOV3; $\mathrm{P}<0.05$, compared with the basal transcriptional level). To address whether the induction of LIV1 transcription gave rise to the upregulated level of LIV1 protein, cultured A2780 and SKOV3 cells were treated with TSA or DMSO and examined for the LIV1 protein using western blotting. As expected, treatment of TSA significantly enhanced the protein levels of LIV1 in the ovarian cancer cells (Fig. 1C and D). Next, to determine whether the TSA-induced expression of LIV1 occurs in primary ovarian cancer cells, 
A

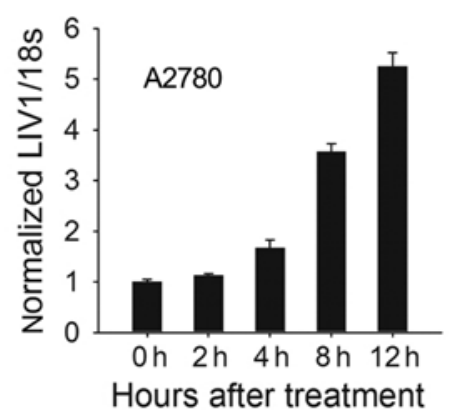

C

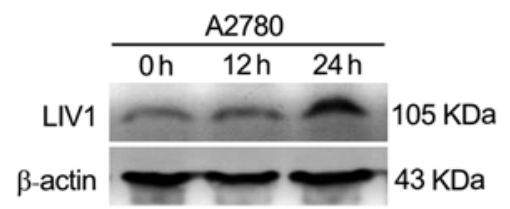

B

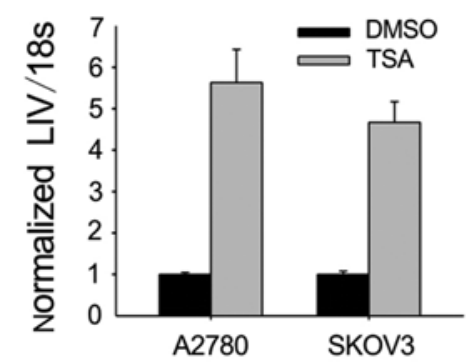

D

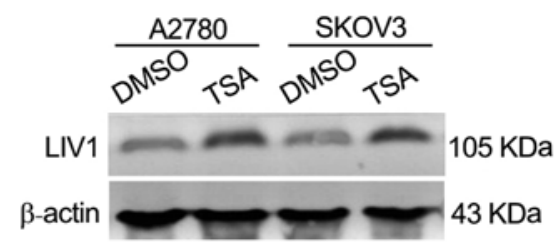

Figure 1. TSA induces the expression of LIV1 in ovarian cancer cells. (A) A2780 cells were treated with 500 nmol/1 TSA for the indicated times and subjected to analysis of real-time PCR for the mRNA levels of LIV1. Results were normalized to those of 18s RNA and are expressed as the fold induction compared with the 0 -h group. The transcriptional level of LIV1 for the $0 \mathrm{~h}$ group was set to $1 .{ }^{*} \mathrm{P}<0.05$, compared with the $0 \mathrm{~h}$ group. (B) A2780 and SKOV3 cells were treated with $500 \mathrm{nmol} / 1$ TSA or DMSO for $12 \mathrm{~h}$ and analyzed for the transcriptional levels of LIV1. (C) A2780 cells were treated with $500 \mathrm{nmol} / 1 \mathrm{TSA}$ for the indicated times and subjected to western blot analysis for the protein levels of LIV1. (D) A2780 and SKOV3 cells were treated with 500 nmol/1 TSA or DMSO for $24 \mathrm{~h}$ and analyzed for the protein levels of LIV1.

Table I. Effect of TSA on the expression of LIV1 in the clinical tumor samples.

\begin{tabular}{|c|c|c|c|c|c|c|}
\hline \multirow[b]{2}{*}{ Patients } & \multirow[b]{2}{*}{ Clinical diagnosis } & \multirow[b]{2}{*}{ Classification } & \multicolumn{4}{|c|}{ Time course $(\mathrm{h})$} \\
\hline & & & 0 & 3 & 6 & 12 \\
\hline Patient 1 & Ovarian cancer & Serous & 1 & $15.3 \pm 3.24$ & $10.16 \pm 2.54$ & $8.75 \pm 2.93$ \\
\hline Patient 2 & Ovarian cancer & Mucinous & 1 & $14.5 \pm 2.83$ & $9.59 \pm 2.19$ & $5.84 \pm 1.45$ \\
\hline Patient 3 & Ovarian cancer & Serous & 1 & $13.2 \pm 3.56$ & $10.96 \pm 2.78$ & $6.57 \pm 1.38$ \\
\hline Patient 4 & Ovarian cancer & Serous & 1 & $9.8 \pm 1.98$ & $8.25 \pm 1.33$ & $7.18 \pm 1.59$ \\
\hline Patient 5 & Ovarian cancer & Mucinous & 1 & $14.1 \pm 3.72$ & $6.41 \pm 1.21$ & $5.66 \pm 1.17$ \\
\hline Patient 6 & Ovarian cancer & Serous & 1 & $10.3 \pm 2.41$ & $8.57 \pm 1.65$ & $6.92 \pm 1.55$ \\
\hline
\end{tabular}

Data are expressed as fold increase (mean \pm SE) of LIV1 mRNA from the TSA-treated cells relative to that from the medium-treated cells. Each data value was analyzed at least in 3 independent experiments.

6 primary tumor samples from patients with ovarian cancer were treated with $500 \mathrm{nmol} / 1 \mathrm{TSA}$ for different time periods. Again, TSA significantly induced the expression of LIV1 at a maximal level (patient 1 for example: $3 \mathrm{~h}, 15.3 \pm 3.24$-fold; $6 \mathrm{~h}$, $10.16 \pm 2.54$-fold; and 12 h, 8.75 \pm 2.93 -fold; $\mathrm{P}<0.05$ ) as early as $3 \mathrm{~h}$ after treatment and the increase lasted up to $12 \mathrm{~h}$ in all of the samples examined (Table I).

Knockdown of LIV1 suppresses cell death induced by TSA in ovarian cancer cells. To confirm whether expression of LIV1 significantly affects TSA-induced cell death in ovarian cancer cells, A2780 and SKOV3 cells were stably transfected with the LIV1 antisense plasmid AS-LIV1 or PCEP4-CAT followed by a 5-day treatment with TSA, and they were then examined for growth inhibition. AS-LIV1 was confirmed to significantly knockdown the basal and TSA-induced levels of LIV1 expression (Fig. 2A), and transfection of AS-LIV1 resulted in the resistance of the cells to TSA treatment (Fig. 2B and C).
Furthermore, we treated the A2780 and SKOV3 cells stably transfected with AS-LIV1 or PCEP4-CAT with a TSA dose range between 300 and $700 \mathrm{nmol} / \mathrm{l}$ for $72 \mathrm{~h}$ and measured the cell viability using the MTT assay. As shown in Fig. 2D and E, knockdown of LIV1 decreased the TSA-induced killing efficiency in both the A2780 and SKOV3 cells at every dose, with a maximum effect observed at a $500 \mathrm{nmol} / 1$ concentration, where the rate of viable cells was increased over $29 \%$ in the A 2780 and $24 \%$ in the SKOV3 cells. In addition, knockdown of LIV1 suppressed TSA- or SAHA (a structurally diverse HDACi)-induced killing and gave rise to more surviving colonies (Fig. 3A and B). Accordingly, knockdown of LIV1 made A2780 and SKOV3 cells resistant to TSA-induced apoptosis (Fig. 3C and D). A2780 and SKOV3 cells stably transfected with AS-LIV1 were much less sensitive to TSA-induced apoptosis than cells stably transfected with PCEP4-CAT (A2780, $18.7 \pm 3.6$ vs. $49.06 \pm 4.3 \%, \mathrm{P}<0.05$; SKOV3, $16.28 \pm 2.9$ vs. $38.13 \pm 3.5 \%, \mathrm{P}<0.05)$. 
A

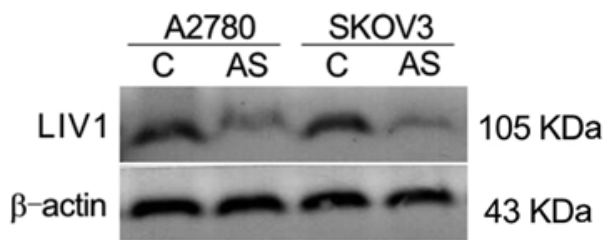

B

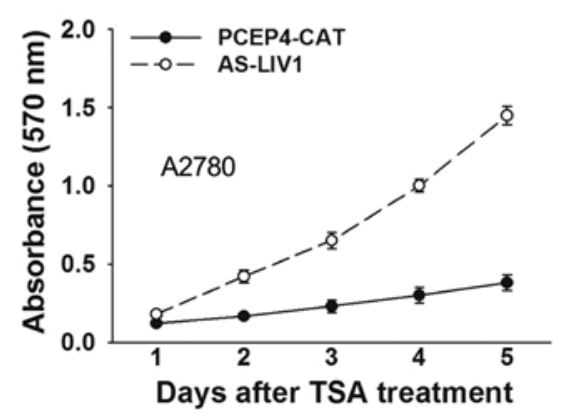

D

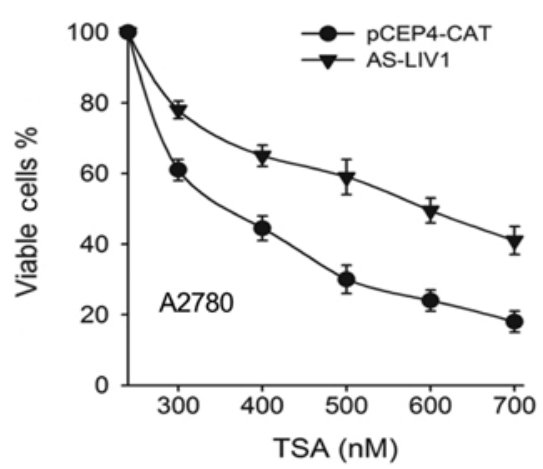

C

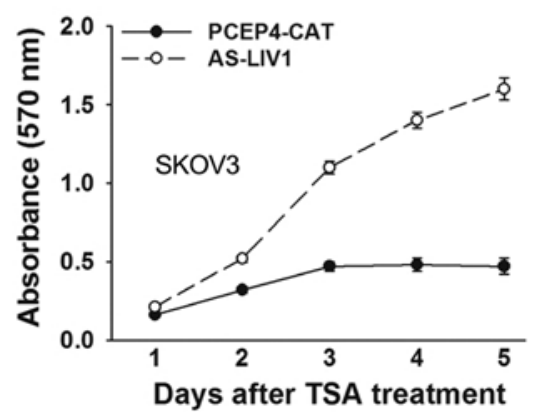

$\mathbf{E}$

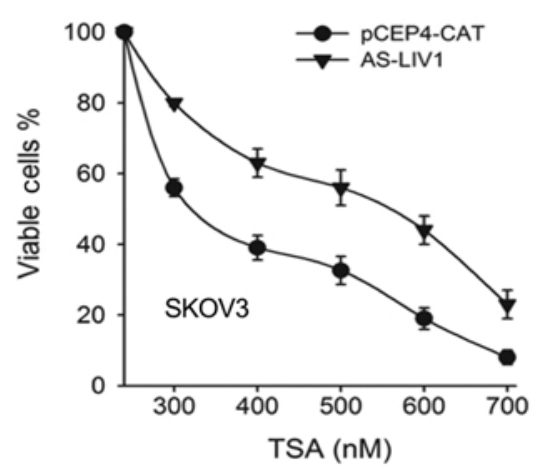

Figure 2. Knockdown of LIV1 debilitates the growth inhibition induced by TSA in ovarian cancer cells. (A) A2780 and SKOV3 cells were stably transfected with AS-LIV1 (AS) and PCEP4-CAT (C). Western blot analysis was used to detect the expression of LIV1. (B) A2780 cells were stably transfected with AS-LIV1 and PCEP4-CAT followed by a 5-day treatment with $500 \mathrm{nmol} / 1 \mathrm{TSA}$. An increase in absorbance indicates cell growth. Data points, mean of five replicates; bars, SEM. (C) SKOV3 cells were treated as in A, and analyzed for cell growth. (D) A2780 and (E) SKOV3 cells were stably transfected with AS-LIV1 and PCEP4-CAT and were exposed to TSA at the indicated concentrations for $72 \mathrm{~h}$. Cell growth was assessed by MTT assay. Results show viable cells in the TSA-treated group and are expressed as a percentage of the viable cells in the DMSO-treated group. Data points, mean of six replicates; bars, SEM.

A

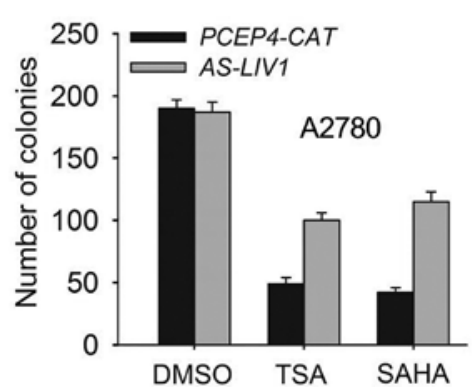

C

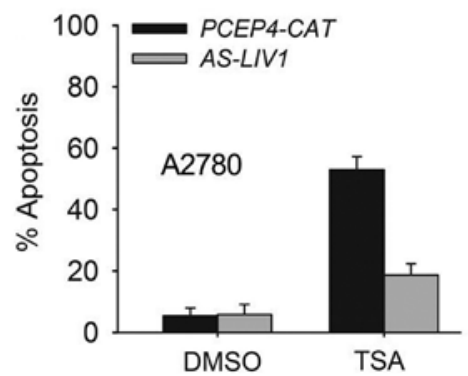

B

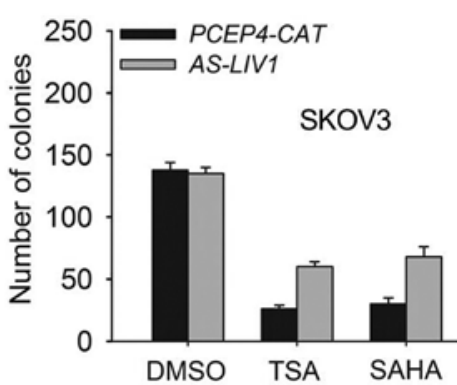

D

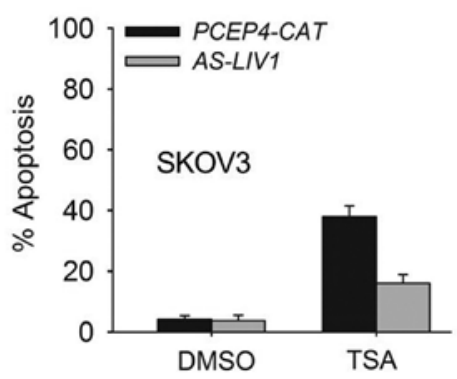

Figure 3. Knockdown of LIV1 suppresses the cell death induced by TSA in ovarian cancer cells. (A) A2780 and (B) SKOV3 cells stably transfected with AS-LIV1 and PCEP4-CAT were treated with $500 \mathrm{nmol} / \mathrm{l} \mathrm{TSA}$ for $24 \mathrm{~h}$, and subjected to colony forming assay; bars, SEM. (C) A2780 and (D) SKOV3 cells stably transfected with AS-LIV1 and PCEP4-CAT were exposed to $1 \mu \mathrm{mol} / 1 \mathrm{TSA}$ for $48 \mathrm{~h}$ and subjected to apoptosis analysis using flow cytometry, where each data point represents the mean \pm SEM of three replicates. 
$\mathbf{A}$

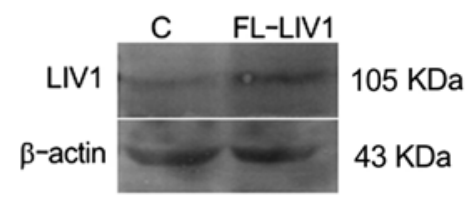

B

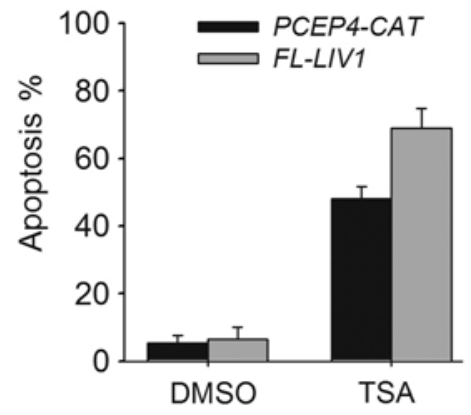

C

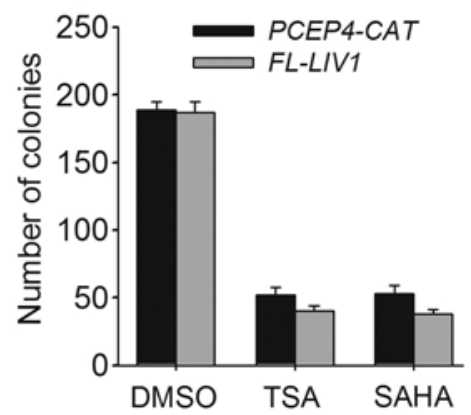

Figure 4. Upregulation of LIV1 enhances cell death induced by TSA in ovarian cancer cells. (A) A2780 cells were stably transfected with FL-LIV1 and PCEP4-CAT (C). Western blot analysis was used to detect the expression of LIV1. (B) A2780 cells stably transfected with FL-LIV1 and PCEP4-CAT were exposed to $1 \mu \mathrm{mol} / 1 \mathrm{TSA}$ for $48 \mathrm{~h}$ and subjected to apoptosis analysis using flow cytometry, where each data point represents the mean \pm SEM of three replicates. (C) A2780 cells stably transfected with FL-LIV1 and PCEP4-CAT were treated with $500 \mathrm{nmol} / 1$ TSA for $24 \mathrm{~h}$, and subjected to coloby formation assay; bars, SEM.

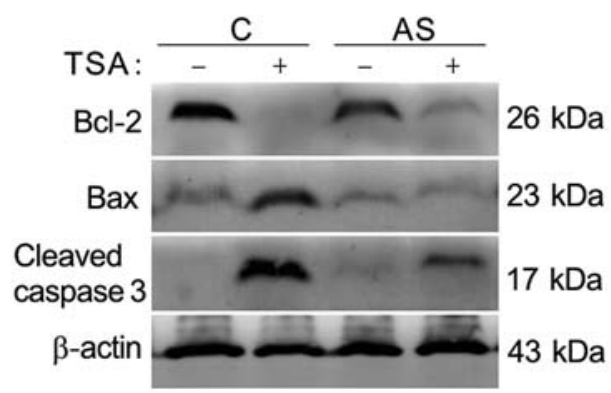

Figure 5. Knockdown of LIV1 protects cells from TSA-induced apoptosis by affecting expression of Bcl-2 family and activity of caspase-3. A2780 cells stably transfected with AS-LIV1 (AS) and PCEP4-CAT (C) were treated with $1 \mu \mathrm{mol} / 1$ TSA or DMSO for $48 \mathrm{~h}$. Western blot analysis was used to detect the expression of Bcl-2, Bax and cleaved caspase-3.

Upregulation of LIVI enhances cell death induced by TSA in ovarian cancer cells. To further verify whether LIV1 is capable of conferring a significant resistance to TSA-induced cell death in ovarian cancer cells from a contrasting perspective, we conducted LIV1 full-length plasmid FL-LIV1, which was confirmed to obviously increase the level of LIV1 expression (Fig. 4A). A2780 cells stably transfected with FL-LIV1 were much more sensitive to TSA-induced apoptosis than cells stably transfected with PCEP4-CAT $(69.02 \pm 4.5$

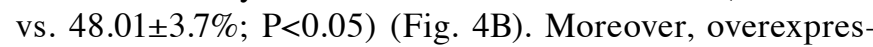
sion of LIV1 promoted TSA- and SAHA-induced cell death and decreased the number of surviving colonies (Fig. 4C). Therefore, it appears that LIV1 modulates the killing efficacy of TSA and may be a critical regulator of cell growth and death in ovarian cancer cells.

Knockdown of LIV1 protects cells from TSA-induced apoptosis by affecting Bcl-2 family and activity of caspase-3. Our previous data suggest that the inhibition of TSA-induced apoptosis by knockdown of LIV1 might be associated with its ability to disrupt intracellular zinc homeostasis (12). Zinc was shown to be a critical regulator of cell growth and death. Previous findings indicate that the mechanistic actions of zinc are shown through change in caspase enzyme activities, as well as the direct alteration of apoptotic regulator expression (16-19). As shown in Fig. 5, TSA induced apoptosis by decreasing endogenous levels of Bcl-2, enhancing levels of Bax and cleavage of procaspase-3. In contrast, the TSA-induced alteration mentioned above could be significantly reversed by LIV-1 knockdown, indicating that LIV1 plays a critical role rather than a by-phenomenon in TSA-mediated apoptosis.

\section{Discussion}

Most patients with ovarian cancer have progressed to advanced stages by the first clinical visit, and are not eligible to be treated with surgery. Thus, these patients can only receive chemotherapy with poor results. Drug resistance is the primary cause of death in late-stage patients. The flood of new second line drugs in recent years has provided many dramatic improvements in anticancer therapy $(20,21)$. Thus, development of new therapeutic strategies and the search for novel genes with new mechanisms of action that lead to drug resistance of ovarian cancer cells have become the focus of current cancer research.

In our previous research, we conducted a functional gene screen approach named suppression of mortality by antisense rescue technique to identify the key genes responsible for the tumor-selective killing of TSA. LIV1 was identified as a critical mediator responsible for TSA-induced apoptosis. LIV1 belongs to a new subfamily of Zrt-, Irt-like protein (ZIP) zinc transporters, now termed the LIV1 subfamily of ZIP zinc transporters (LZT) (22). Based on its amino acid sequence and its cellular location on the plasma membrane, it has been proposed as a putative zinc transporter involved in maintaining intracellular zinc homeostasis (23). Previous investigations have demonstrated that LIV1 expression is associated with 
small estrogen receptor-positive tumors of which $92 \%$ show lymph node involvement, and its expression may be both a suitable prognostic marker for lymph node involvement and metastatic spread in steroid hormone receptor-positive disease (24). In breast cancer, high LIV1 protein expression is associated with a better clinical outcome in patients with breast cancer. In zebrafish gastrula organizer, LIV1 controls epithelial-mesenchymal transition. Nevertheless, the biological function of the LIV1 gene is still not well understood. Our findings presented here highlight an essential role for LIV1 in drug resistance in ovarian cancer cells which might extend our understanding of LIV1 in the regulation of apoptosis in cancer.

The present study confirmed that LIV1 expression is obviously induced by TSA treatment in ovarian cancer cells. Knockdown of LIV1 protects ovarian cancer cells from TSA-induced apoptosis and it is associated with the alteration of activity of caspase-3 and the Bcl-2 family. It is well-known that the caspase family is a cysteine protease family, among which the proteolysis cascade reaction controls the development of cell apoptosis. Caspase-3 is related to several events during the effector phase of apoptosis and its activation serves as a common channel for apoptosis pathways $(25,26)$. This experiment confirmed that knockdown of LIV1 could significantly decrease activated casepase-3, eventually leading to irreversible drug resistance. Furthermore, programmed cell death is a well-orchestrated process regulated by multiple pro-apoptotic and anti-apoptotic genes, particularly those of the $\mathrm{Bcl}-2$ gene family. $\mathrm{Bcl}-2$ is an integral membrane protein located mainly on the outer membrane of mitochondria. Overexpression of $\mathrm{Bcl}-2$ prevents cells from undergoing apoptosis in response to a variety of stimuli. In contrast, Bax promotes apoptosis $(27,28)$. Our data are also consistent with previous findings that knockdown of LIV1 increased Bcl-2 expression and decreased Bax expression. Taken together, our findings have identified LIV1 as a novel target responsible for sensivity of ovarian cancer cells to TSA. The novel mechanism proposed here might have important clinical potential. Given that LIV1 is a novel identified gene with undefined functions, further animal experiments and clinical studies are needed to determine their therapeutic effects in vivo, and further characterization of LIV1 would aid in the development of more effective protocols.

\section{Acknowledgements}

The present study was supported by a grant from the National Natural Science Foundation of China (no. 81101970); Ph.D. Programs Foundation of the Ministry of Education of China (no. 20111107120009); and the Scientific Research Common Program of Beijing Municipal Commission of Education (KM 201210025020).

\section{References}

1. Jemal A, Bray F, Center MM, et al: Global cancer statistics. CA Cancer J Clin 61: 69-90, 2011.

2. Hennessy BT, Coleman RL and Markman M: Ovarian cancer. Lancet 374: 1371-1382, 2009.

3. Zahedi P, Yoganathan R, Piquette-Miller M and Allen C: Recent advances in drug delivery strategies for treatment of ovarian cancer. Expert Opin Drug Deliv 9: 567-583, 2012.
4. Vecchione A, Belletti B, Lovat F, et al: A microRNA signature defines chemoresistance in ovarian cancer through modulation of angiogenesis. Proc Natl Acad Sci USA 110: 9845-9850, 2013.

5. West AC and Johnstone RW: New and emerging HDAC inhibitors for cancer treatment. J Clin Invest 2: 30-39, 2014.

6. Slingerland M, Guchelaar HJ and Gelderblom H: Histone deacetylase inhibitors: an overview of the clinical studies in solid tumors. Anticancer Drugs 25: 140-149, 2014.

7. Højfeldt JW, Agger K and Helin K: Histone lysine demethylases as targets for anticancer therapy. Nat Rev Drug Discov 12: 917-930, 2013.

8. Marchion D and Munster P: Development of histone deacetylase inhibitors for cancer treatment. Expert Rev Anticancer Ther 7: 583-598, 2007.

9. Marks PA and Breslow R: Dimethyl sulfoxide to vorinostat: development of this histone deacetylase inhibitor as an anticancer drug. Nat Biotechnol 25: 84-90, 2007.

10. Rasheed WK, Johnstone RW and Prince HM: Histone deacetylase inhibitors in cancer therapy. Expert Opin Investig Drugs 16: 659-678, 2007.

11. Fakih MG, Pendyala L, Fetterly G, et al: A phase I, pharmacokinetic and pharmacodynamic study on vorinostat in combination with 5-fluorouracil, leucovorin, and oxaliplatin in patients with refractory colorectal cancer. Clin Cancer Res 15: 3189-3195, 2009.

12. Ma XL, Ma QF, Liu J, et al: Identification of LIV1, a putative zinc transporter gene responsible for HDACi-induced apoptosis, using a functional gene screen approach. Mol Cancer Ther 8: 3108-3116, 2009.

13. Yamashita S, Miyagi C, Fukada T, et al: Zinc transporter LIV-1 controls epithelial-mesenchymal transition in zebrafish gastrula organizer. Nature 429: 298-302, 2004.

14. Kasper G, Weiser AA, Rump A, et al: Expression levels of the putative zinc transporter LIV-1 are associated with a better outcome of breast cancer patients. Int J Cancer 117: 961-973, 2005.

15. Ma XL, Liu J, Wu J, et al: Synergistic killing effect between vorinostat and target of CD146 in malignant cells. Clin Cancer Res 16: 5165-5176, 2010.

16. Sankavaram K, Chong L, Bruno RS and Freake HC: Zinc status alters growth and oxidative stress responses in rat hepatoma cells. Nutr Cancer 66: 104-116, 2014.

17. Nygaard SB, Larsen A, Knuhtsen A, et al: Effects of zinc supplementation and zinc chelation on in vitro $\beta$-cell function in INS-1E cells. BMC Res Notes 7: 84, 2014.

18. Zhang X, Zhao Y, Chu Q, et al: Zinc modulates high glucoseinduced apoptosis by suppressing oxidative stress in renal tubular epithelial cells. Biol Trace Elem Res 158: 259-267, 2014.

19. Chimienti F, Aouffen M, Favier A and Seve M: Zinc homeostasisregulating proteins: new drug targets for triggering cell fate. Curr Drug Targets 4: 323-338, 2003.

20. Jayson GC, Kohn EC, Kitchener HC and Ledermann JA: Ovarian cancer. Lancet 13: 62146-62147, 2014.

21. Marcus CS, Maxwell GL, Darcy KM, et al: Current approaches and challenges in managing and monitoring treatment response in ovarian cancer. J Cancer 5: 25-30, 2014.

22. Taylor KM and Nicholson RI: The LZT proteins; the LIV-1 subfamily of zinc transporters. Biochim Biophys Acta 1611: 16-30, 2003.

23. Taylor KM: LIV-1 breast cancer protein belongs to a new family of histidine-rich membrane proteins with potential to control intracellular $\mathrm{Zn}^{2+}$ homeostasis. IUBMB Life 49: 294-253, 2000.

24. Taylor KM, Morgan HE, Johnson A, et al: Structure-function analysis of LIV-1, the breast cancer-associated protein that belongs to a new subfamily of zinc transporters. Biochem J 375: 51-59, 2003

25. Hensley P, Mishra M and Kyprianou N: Targeting caspases in cancer therapeutics. Biol Chem 394: 831-843, 2013.

26. Maghsoudi N, Zakeri Z and Lockshin RA: Programmed cell death and apoptosis-where it came from and where it is going: from Elie Metchnikoff to the control of caspases. Exp Oncol 34: 146-152, 2012.

27. Ola MS, Nawaz M and Ahsan $\mathrm{H}$ : Role of Bcl-2 family proteins and caspases in the regulation of apoptosis. Mol Cell Biochem 351: 41-58, 2011.

28. Thomas S, Quinn BA, Das SK, et al: Targeting the Bcl-2 family for cancer therapy. Expert Opin Ther Targets 17: 61-75, 2013. 